\title{
Stay-green protein, defective in Mendel's green cotyledon mutant, acts independent and upstream of pheophorbide $a$ oxygenase in the chlorophyll catabolic pathway
}

\author{
Sylvain Aubry · Jan Mani · Stefan Hörtensteiner
}

Received: 18 October 2007/ Accepted: 12 February 2008/Published online: 27 February 2008

(C) Springer Science+Business Media B.V. 2008

\begin{abstract}
Type C stay-green mutants are defined as being defective in the pathway of chlorophyll breakdown, which involves pheophorbide $a$ oxygenase (PAO), required for loss of green color. By analyzing senescence parameters, such as protein degradation, expression of senescenceassociated genes and loss of photosynthetic capacity, we demonstrate that J2775, the green cotyledon $(i)$ pea line used by Gregor Mendel to establish the law of genetics, is a true type C stay-green mutant. STAY-GREEN (SGR) had earlier been shown to map to the $I$ locus. The defect in JI2775 is due to both reduced expression of SGR and loss of SGR protein function. Regulation of PAO through SGR had been proposed. By determining PAO protein abundance and activity, we show that PAO is unaffected in JI2775. Furthermore we show that pheophorbide $a$ accumulation in the mutant is independent of PAO. When silencing $S G R$ expression in Arabidopsis paol mutant, both pheophorbide $a$ accumulation and cell death phenotype, typical features of paol, are lost. These results confirm that
\end{abstract}

Electronic supplementary material The online version of this article (doi:10.1007/s11103-008-9314-8) contains supplementary material, which is available to authorized users.

S. Aubry $\cdot$ S. Hörtensteiner $(\bowtie)$

Institute of Plant Biology, University of Zurich,

Zollikerstrasse 107, 8008 Zurich, Switzerland

e-mail: shorten@botinst.uzh.ch

J. Mani · S. Hörtensteiner

Institute of Plant Sciences, University of Bern, Altenbergrain 21,

3013 Bern, Switzerland

Present Address:

J. Mani

Institute of Cell Biology, University of Bern, Baltzerstr. 4,

3012 Bern, Switzerland
SGR function within the chlorophyll catabolic pathway is independent and upstream of PAO.

Keywords Chlorophyll breakdown - Gregor Mendel . Pea $I$ locus · Pheophorbide $a$ oxygenase .

Stay-green mutant $\cdot$ Senescence

$\begin{array}{ll}\text { Abbreviations } \\ \text { chl } & \text { Chlorophyll } \\ \text { chlide } & \text { Chlorophyllide } \\ \text { EST } & \text { Expressed sequence tags } \\ \text { LHCII } & \text { Light harvesting complex of photosystem II } \\ \text { NCCs } & \text { Nonfluorescent chlorophyll catabolites } \\ \text { pheide } & \text { Pheophorbide } \\ \text { PAO } & \text { Pheophorbide } a \text { oxygenase } \\ \text { pFCC } & \text { Primary fluorescent chlorophyll catabolite } \\ \text { PS } & \text { Photosystem } \\ \text { RCCR } & \text { Red chlorophyll catabolite reductase } \\ \text { Sgr } & \text { Stay-green }\end{array}$

\section{Introduction}

Chlorophyll (chl) breakdown is widely used as a tool for monitoring physiological senescence in plants. Thereby green chl is converted in a multistep pathway to linear colorless and nonfluorescent tetrapyrroles, NCCs. The pathway starts with the consecutive removal from chl of phytol and the $\mathrm{Mg}$ atom by chlorophyllase and a metalchelating substance, respectively. Loss of the green color occurs during the subsequent opening of the porphyrin macrocycle of pheophorbide (pheide) $a$ by the coupled action of pheide $a$ oxygenase (PAO) and red chl catabolite reductase (RCCR). This yields a primary fluorescent 
catabolite (pFCC), which is exported from the plastid by a primary active transporter. Further steps in the pathway are reminiscent of detoxification processes widely occurring in plants (Kreuz et al. 1996). Thus, pFCCs are activated by hydroxylation and after further modification they are imported into the vacuole by another primary active transport system. Due to the acidic vacuolar $\mathrm{pH}$, FCCs eventually tautomerize non-enzymatically to NCCs (Oberhuber et al. 2003; Hörtensteiner 2006).

All but one of the NCCs isolated so far from higher plants have been shown to derive from chl $a$ (Hörtensteiner 2006; Müller et al. 2006). One reason for this is the substrate-specificity of PAO for pheide $a$, with pheide $b$ being a competitive inhibitor (Hörtensteiner et al. 1995). Furthermore, reduction of chl $b$ to chl $a$ within the chl-protein complexes of the photosystems (PS), has been suggested a prerequisite for chl degradation, leading to the destabilization of these chl-protein complexes (Hörtensteiner 2006). The recent cloning of NYC1 from rice (Kusaba et al. 2007) corroborated this assumption. nycl mutants show a staygreen phenotype and besides the retention of both chl $a$ and $b$, nycl specifically retains light harvesting complex of photosystem II (LHCII) subunits known to bind large proportions of chl $b$. In this respect NYC1 represent an early (primary) element of metabolic regulation of chl breakdown.

Another step of regulation is at the level of PAO. Like NYC1, PAO expression is senescence-specific and absence of PAO in a respective mutant, paol, leads to a stay-green phenotype in the dark. Due to the accumulation of phototoxic pheide $a$, paol plants show a light-dependent lesion mimic phenotype (Pružinská et al. 2005).

Recently, a third type of stay-green mutants has become a major focus in plant senescence research. These mutants are defective in a gene identified in the Bf993 mutant of Festuca pratensis (Thomas 1987). The gene was termed SID (senescence-induced degradation), but orthologous genes from rice, pea and Arabidopsis have now been designated SGR (STAY-GREEN) (Jiang et al. 2007; Park et al. 2007; Sato et al. 2007), SGN (Park et al. 2007) or NYE1 (nonyellowing) (Ren et al. 2007). Introgression of the sid locus into different Lolium species allowed the molecular tagging of the gene (Thomas 1997). By exploiting the high micro synteny between Lolium-Festuca and rice, it was possible to identify the candidate locus in a Lolium staygreen mapping population that corresponded to a staygreen locus $(S G R)$ in rice (Armstead et al. 2006). Silencing of the orthologous gene, AtSGRl (At4 g22920) of Arabidopsis, caused the same stay-greenness as in Festuca or Lolium (Armstead et al. 2007).

Furthermore, it has been shown that $S G R$ maps to the $I$ locus of Mendel's green cotyledon mutant (Armstead et al. 2007). Thus, the $I$ locus is only the third identified locus of the seven traits Gregor Mendel used for establishing his laws of genetic inheritance (Mendel 1866). The first locus that has been molecularly identified is the so-called $r$ (rugosus) locus. The mutation, which is due to a $0.8 \mathrm{~kb}$ insertion, resides in a gene encoding a starch branching enzyme and causes the formation of wrinkled seeds (Bhattacharyya et al. 1990). The second identified gene (Le locus), encodes gibberellin $3 \beta$-hydroxylase. The loss of function missense mutation near the active site abolishes gibberellin biosynthesis and, thus, affects stem length (Lester et al. 1997). Recently, the mutation of Mendel's I locus was elucidated molecularly (Sato et al. 2007). In contrast to an earlier finding of reduced $S G R$ gene expression in the mutant (Armstead et al. 2007), gene expression was found to be normal. By complementing a rice $s g r$ mutant with rice $S G R$ gene exhibiting a 6-bp-insertion, it was concluded that the analogous mutation present in Mendel's green pea $S G R$ gene abolished SGR function (Sato et al. 2007).

The Festuca sid mutant is a classical representative of the so-called 'cosmetic' type C stay-green mutants (Thomas and Howarth 2000). In these non-functional stay-greens, retention of chl is uncoupled from other senescence events, such as loss of photosynthetic capacity, which proceed normally (Hilditch et al. 1986, 1989). Hence, the gene mutations of type $\mathrm{C}$ mutants were considered to affect the pathway of chl breakdown directly. This has been confirmed and is obvious for paol and nycl, which are affected in catabolic steps of the pathway (Pružinská et al. 2003, 2005; Kusaba et al. 2007). The situation is different for $s g r /$ sid mutants, since so far the function of SGR/SID could not unequivocally be demonstrated. Several early reports deal with the biochemical lesion of sid and sid-like mutants, including Mendel's I mutant (Vicentini et al. 1995; Thomas et al. 1996; Roca et al. 2004). The cumulative results of these investigations indicated that SGR/SID affects PAO activity. Thus, the mutants consistently accumulated chlorophyllide (chlide) and pheide $a$ during senescence and they exhibited reduced PAO activities. It was argued that pheide $a$ accumulation would feed back inhibit chl breakdown, in order to prevent further chl intermediate accumulation, thereby minimizing the phototoxic potential of these pigments (Thomas 1997; Matile et al. 1999; Hörtensteiner 2006). Along with the retention of chl, chl-binding proteins were shown to be retained in the mutants (Hilditch et al. 1989; Jiang et al. 2007; Park et al. 2007; Sato et al. 2007). Recently, it was demonstrated that rice SGR is able to specifically interact with LHCII subunits (Park et al. 2007). This together with the retention of these proteins in the mutants, suggested that SGR might be involved in dismantling chl-protein complexes as a prerequisite for both chl and apoprotein degradation.

The aim of this study was to thoroughly characterize Mendel's pea mutant (JI2775) in respect to senescence and 
chl breakdown. We confirm that JI2775 is a classical type $\mathrm{C}$ stay-green mutant by analyzing different parameters of senescence, such as chl fluorescence and degradation, expression of senescence associated genes and protein degradation. We further show that JI2775 is impaired not only in SGR protein function, but by $S G R$ expression and SGR immunoblot analysis demonstrate that gene expression is affected as well in the mutant. We re-addressed the proposed linkage between SGR and PAO. Using PAO activity assays and immunoblot analysis, we show that PAO function is unaffected in JI2775. Furthermore, we show that the amount of pheide $a$ accumulating in the mutant is independent of the activity of PAO. Silencing of $S G R$ expression in paol, results in the losses of pheide $a$ accumulation and cell death phenotype, typical features of paol. We conclude that the function of SGR is upstream and independent of PAO. This together with the previously demonstrated interaction of SGR with LHCII, and retention during senescence of $s g r$ mutants of chl binding proteins indicates a (predominant) role of SGR in dismantling of chl-protein complexes during senescence.

\section{Experimental procedures}

Plant material and senescence induction

Pisum sativum lines JI4 (yellow cotyledons) and JI2775 (green cotyledons) were obtained from the Pisum germplasm collection of the John Innes Center, Norwich, UK. The Columbia (Col-0) ecotype of Arabidopsis (Arabidopsis thaliana) was used as wild type. For silencing of AtSGRl by RNA interference, a pHannibal-derived construct as described in Armstead et al. (2007) was used. paol was transformed by a floral dip method and homozygous paol atsgrli lines selected by kanamycin resistance. This was possible, since paol is not resistant to kanamycin (Alonso et al. 2003; Pružinská et al. 2005). Production of the AtSGRl-silencing lines, atsgrli, has been described (Armstead et al. 2007). A homozygous AtSGR2 T-DNA insertion line of the SALK collection (Alonso et al. 2003) was obtained from the European Arabidopsis Stock Center, Nottingham, UK. T-DNA insertion was verified by sequencing a PCR product obtained with the left T-DNA border primer LBb1 (5'-GCGTGGACCGCTTGCTGCAA T-3') and an AtSGR2-specific primer (5'-GCAACTGGT GAAAGCAAGAAC-3'). A Lolium line carrying a sid gene introgression has been described (Roca et al. 2004) and was obtained from H. Ougham, IGER, Aberystwyth, Wales, UK.

Plants were grown on soil in long-day condition $(16 \mathrm{~h} / 8 \mathrm{~h})$

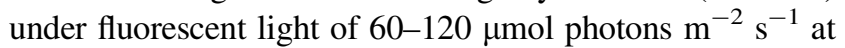
$22^{\circ} \mathrm{C}$. For senescence induction, leaves from 3 - to 4 -week-old plants were excised and incubated in permanent darkness on wet filter paper for up to 11 days at ambient temperature. Alternatively, attached leaves were individually covered with aluminum foil.

\section{Cloning strategy}

Genomic DNA and cDNA was isolated from both JI4 and JI2775 leaves as described (Murray and Thompson 1980; Pružinská et al. 2005). PsSGR was amplified with primers PsSGR-LP (5'-CGGGATCCATGGATACTCTAACGAG TGCTC- $\left.3^{\prime}\right)$ and PsSGR-RP (5'-TTCTGCAGTTACAAGTT ACCATGTTGGGTTC- $3^{\prime}$ ) using the proofreading Pfu polymerase (Promega). After digestion with BamHI and PstI, fragments were cloned into pQE30 (Qiagen) to yield pQEJI4 and pQEJI2775, and inserts sequenced. For constructing binary plasmids, SGR open reading frames were amplified from both pQE plasmids with PsSGR-LP2 (5'-GT ACCATGGATACTCTAACGAGTGCTCCTTTACTC-3') and PsSGR-RP2 (5'-GCGGATCCTTACAAGTTACCATG TTGGGTTC- $3^{\prime}$ ) and, after restriction with $N c o$ I and BamHI cloned into pHannibal (Wesley et al. 2001) containing the CaMV 35S-double enhancer promoter and a nos terminator. The gene constructs were excized by NotI and cloned into pGreen0029 (Hellens et al. 2000) yielding pGr-JI4-SGR and pGr-JI2775-SGR, respectively. After verifying inserts by sequencing, constructs were transformed into Agrobacterium together with pSOUP (Hellens et al. 2000).

\section{Plant transformation}

For transient expression in Nicotiana benthamiana leaves, $5 \mathrm{ml}$ of Agrobacterium harboring the respective plasmids were grown overnight at $28^{\circ} \mathrm{C}$. Cells were collected by centrifugation $(3,000 \mathrm{~g}, 15 \mathrm{~min})$, pellets resuspended in $1 \mathrm{ml}$ of acetosyringone buffer $(1 \mathrm{mM} \mathrm{MgCl} 2,150 \mathrm{mM}$ acetosyringone, $1 \mathrm{mM}$ Mes- $\mathrm{KOH} \mathrm{pH} 5.6$ ) and grown again for $4 \mathrm{~h}$ (Llave et al. 2000). Using a $1 \mathrm{ml}$ syringe, the abaxial side of leaves of 5-week-old $N$. benthamiana plants was infiltrated with the Agrobacterium cultures. For transformation of Arabidopsis paol with a pHannibalderived AtSGR1 silencing construct (Armstead et al. 2007), the floral dip method was used (Sidler et al. 1998).

RNA isolation and real-time PCR

For RT-PCR analysis, total RNA was prepared from Pisum leaves and reverse transcribed as described (Pružinská et al. 2005). Quantitative PCR was performed in a LightCycler (Roche Diagnostics) using the QuantiTect SYBR Green PCR kit (Qiagen). 10- to 100-fold dilutions of firststrand cDNA reaction mixes (corresponding to $0.3-3 \mathrm{ng}$ of RNA) were employed in $20 \mu \mathrm{l}$ reactions and were used to 
calculate the real-time PCR efficiency of each sample. The relative expression ratios of target gene (SGR) were calculated in comparison to a reference gene, $A C T 2$ (Kürsteiner et al. 2003). For semiquantitative and/or quantitative PCR the following specific primers were used: PSACT2 (forward, $5^{\prime}$-TGGAATCCACGAGACAACCTA- $3^{\prime}$ and reverse, $5^{\prime}$-T TCTGTGAACGATTCCTGGAC- $3^{\prime}$ ); $P s S G R$ (forward, $5^{\prime}$ ACGGTGACGGCAATTTATTC- $3^{\prime}$ and reverse, $5^{\prime}$-TTCA ACGGTGGAAAACAACA- $3^{\prime}$ ); PsSAG12 (forward, $5^{\prime}$-TC TGAGCAAGAGCTTGTGGA- $3^{\prime}$ and reverse $5^{\prime}$-TATCCCC CAGCATCAATAGC- $3^{\prime}$ ); AtSGRl (forward, 5'-TGGGCAA ATAGGCTATACCG- $3^{\prime}$ and reverse, $5^{\prime}$-AAGTTCCCATC TCCATGCAC-3'); AtSGR2 (forward, 5'-TCCAACATCC CGGAGTAC- $3^{\prime}$ and reverse, $5^{\prime}$-AGGGTTAGGTATTTCC AAC- $3^{\prime}$ ); ACT2 (forward, 5'-TGGAATCCACGAGACA ACCTA- $3^{\prime}$ and reverse, $5^{\prime}$-TTCTGTGAACGATTCCTG GAC- $\left.3^{\prime}\right)$.

\section{Southern blot analysis}

High molecular weight genomic DNA was isolated from both Pisum lines (Chakraborti et al. 2006). Each $15 \mu \mathrm{g}$ was digested with restriction endonucleases, electrophoresed on $0.9 \%$ agarose gels and transferred to positively charged nylon membrane (Roche). The blot was hybridized overnight at $42^{\circ} \mathrm{C}$ with a digoxigenin-UTP-labeled $1.5 \mathrm{~kb}$ PCR fragment (using pQEJI4 and primers PsSGR-RP and PsSGR-LP) and washed at $65^{\circ} \mathrm{C}$ with $0.2 \times \mathrm{SSC}, 0.1 \%$ SDS. Subsequent detection by anti-digoxigenin-AP (Roche), and bromochloroindolyl phosphate/nitroblue tetrazolium as substrate was according to the manufacturer's protocol.

\section{Analysis of chl and chl catabolites}

Chl was isolated from dark-incubated Arabidopsis and pea leaves, or from $N$. benthamiana according to Pružinská et al. (2005) and chl concentrations were determined spectrophotometrically (Strain et al. 1971). Chl and green pigments (chlide and pheide) were extracted and analyzed as described (Pružinská et al. 2005). For bipyridyl treatments, leaf discs were senesced in the dark by floating on water, $1 \%$ DMSO or

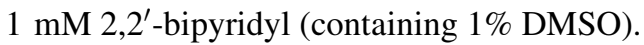

PAO protein extraction and activity measurement

PAO was isolated from Pisum chloroplast membranes by Triton X-100 solubilization according to a standard procedure (Hörtensteiner et al. 1995; Pružinská et al. 2005; Ren et al. 2007). PAO activity was monitored in vitro essentially as described (Pružinská et al. 2007) but using $0.06 \mu \mathrm{g} \mu \mathrm{l}^{-1}$ of $\mathrm{His}_{6}$-AtRCCR (Pružinská et al. 2007), and $100 \mathrm{U}$ of catalase (Sigma) to replace red chl catabolite-forming factor (Pružinská et al. 2005). pFCC formation was followed by reversed-phase HPLC and fluorescence detection (320/ $450 \mathrm{~nm})$ using $36 \%(\mathrm{v} / \mathrm{v}) 50 \mathrm{mM}$ potassium phosphate buffer, pH 7, in methanol as solvent (Pružinská et al. 2005).

Production of anti-AtSGR antiserum and immunoblot analysis

A polyclonal anti-AtSGR antiserum was obtained from rabbits immunized with two polypeptides directed against two AtSGR peptides $\left(\mathrm{NH}_{2}\right.$-EGVNGYSGTQTEGIAT$\mathrm{COOH}$ and $\mathrm{NH}_{2}$-SQSINNSQLQGWAN-COOH). The serum was purified by affinity purification and tested by ELISA (Eurogentec, Seraing, Belgium).

After separation by SDS-PAGE, proteins were transferred to nitrocellulose membranes according to standard procedures. Proteins were labeled with monoclonal antibodies against PAO (1:500; Gray et al. 2004) and polyclonal antibodies against the following proteins: AtSGR $(1: 1,000)$, LHCII (1:2,000; Pružinská et al. 2003), Rubisco small subunit (1:2,000; S. Gepstein, Israel), Rubisco LS (1:2,000; S. Gepstein, Israel), PsaA (1:5,000; J.D. Rochaix, Geneva, Switzerland), PsaD (1:5,000; J.D. Rochaix, Geneva, Switzerland), Lhca (1:2,000; AgriSera, Sweden), PsbA (1:1,000; H. Ougham, Aberystwyth, Wales), and Cytf (1:1,000; H. Ougham, Aberystwyth, Wales). Subsequently, the membranes were labeled with alkaline phosphatase- or peroxidase-conjugated secondary antibodies, and visualized using bromochloroindolyl phosphate/nitroblue tetrazolium (PsbA and Cytf), or with the chemiluminescent peroxidase substrate kit (Sigma). Chemiluminescence signals were quantified using an ImageQuant 400 system (GE Healthcare). Immunoblot analyses were carried out in duplicate or triplicate.

Chlorophyll fluorescence and $\mathrm{CO}_{2}$ assimilation rate

Senescence was induced by darkening attached leaves with aluminum foil. Using a portable photosynthesis system LI-COR 6400 (Li-Cor, Lincoln, NE), maximum quantum yield of PSII $\left(F_{\mathrm{v}} / F_{\mathrm{m}}\right)$ and minimal chl $a$ fluorescence following dark incubation $\left(F_{0}\right)$ were determined. Quantum efficiency of electron transport through PSII (ФPSII) was measured after adaptation to day light for $20 \mathrm{~min}$ (Maxwell and Johnson 2000). Rates of $\mathrm{CO}_{2}$ assimilation were determined at a flux density of $500 \mu \mathrm{mol} \mathrm{m} \mathrm{m}^{-2} \mathrm{~s}^{-1}$ and a $\mathrm{CO}_{2}$ concentration of $380 \mu \mathrm{mol} \mathrm{mol}{ }^{-1}$.

SGR protein accession numbers

The GenBank accession numbers of SGRs and SGR-like proteins in plants, algae and bacteria species are as follows: Arabidopsis thaliana AtSGR1, AAW82962; AtSGR2, AAU05981; AtSGR3, AAM14392; Bacillus sp. BsSGR1, 
ZP_01859452; BsSGR2， ZP_01170511; Clostridium botulinum CbSGR, CAL83726; Clostridium purfigens CpSGR, Q8XH99; Glycine max GmSGR1, AAW82959; GmSGR2, AAW82960; Hordeum vulgare HvSGR, AAW82955; Lycopersicon esculentum LeSGR, AAY98500; Oryza sativa OsSGR1, AAW82954; OsSGR2, BAF16284; OsSGR3, CAE05787; Ostreococcus lucimarinus OlSGR, XP_001420948; Ostreococcus tauri OtSGR, CAL56489; Pisum sativum PsSGR-JI4, CAP04954; PsSGRJI2775, CAP04955; Sorghum bicolor SbSGR, AAW82958; Vitis vinifera VvSGR1, CAO63641; VvSGR2, CAO63642; VvSGR3, CAN81476; Zea mays ZmSGR1, AAW82956; ZmSGR2, AAW82957; Zoysia japonica ZjSGR, AAW82961. The following protein sequences were obtained from the Joint Genome Institute (http://jgi.doe.gov) (protein IDs are given) Populus trichocarpa PtSGR1, 548540; PtSGR2, 646534; Chlamydomonas reinhardtii CrSGR1, 144728; CrSGR2, 168164; Physcomitrella patens PpSGR1, 128024; PpSGR2, 139487; PpSGR3, 114221; PpSGR4, 27214.

\section{Results and discussion}

Mendel's $i$ mutant exhibits a non-functional stay-green phenotype

Gregor Mendel's green cotyledon mutant, JI2775 (i/i), was compared with JI4 (I/I), a wild type line showing normal yellowing of seeds during maturation (Fig. 1a) (White 1916). JI4 and JI2775 are non-isogenic lines and exhibit some differences in respect to morphology, like a difference in average size of mature leaves (JI4: $12.63 \pm 1.24 \mathrm{~cm}^{2}$; JI2775: $8.33 \pm 1.25 \mathrm{~cm}^{2}$ ), or seed weight (JI4: $284 \pm$ $30.5 \mathrm{mg}$; J12775: $212.25 \pm 39 \mathrm{mg}$ ). The stay-green phenotype of JI2775 was evident in all green parts of the plants, i.e. besides seeds and leaves (Fig. 1a) also in pods and stems (not shown).

To induce senescence, detached leaves were incubated in permanent darkness (Fig. 1a). After 8d dark incubation, $84 \%$ of chl was retained in JI2775, thus showing a stronger retention than stay-green Lolium temulentum (Roca et al. 2004) or Arabidopsis nyel (Ren et al. 2007). Increase in the chl $a$ to $b$ ratio is a characteristic of senescence-related chl breakdown (Pružinská et al. 2005). This is caused by chl $b$ to $a$ reduction, which is a prerequisite of breakdown beyond the level of pheide (Scheumann et al. 1999). Consequently, the chl $a: b$ ratio increased during dark-incubation of JI4 leaves, but not in J12775, indicating that both chl $a$ and $b$ are retained to the same extend in the mutant (Fig. 1c).

Stay-greenness during senescence does not necessarily imply a conservation of photosynthetic capacity, as seen in other instances of non-functional or so called cosmetic stay-
(A)
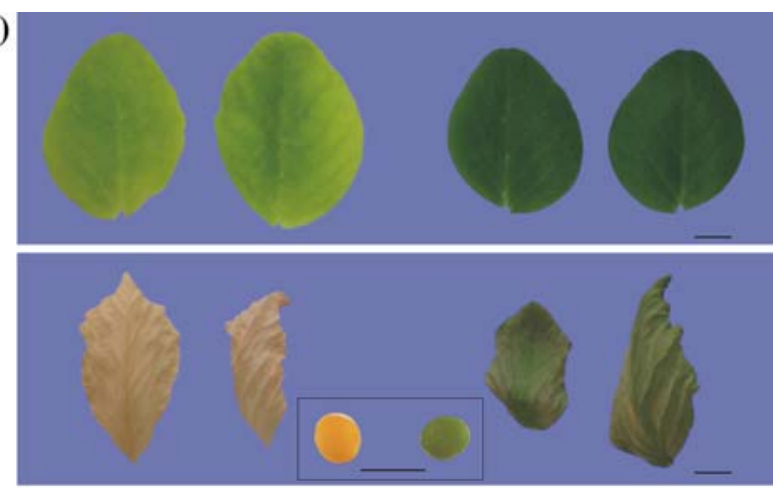

(B)

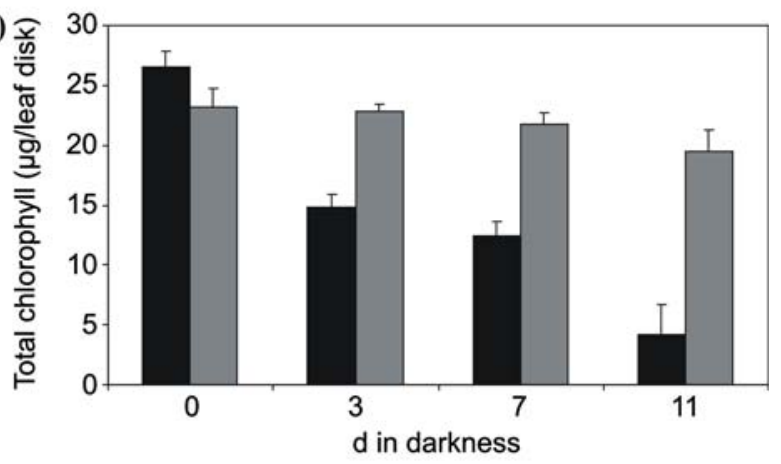

(C)

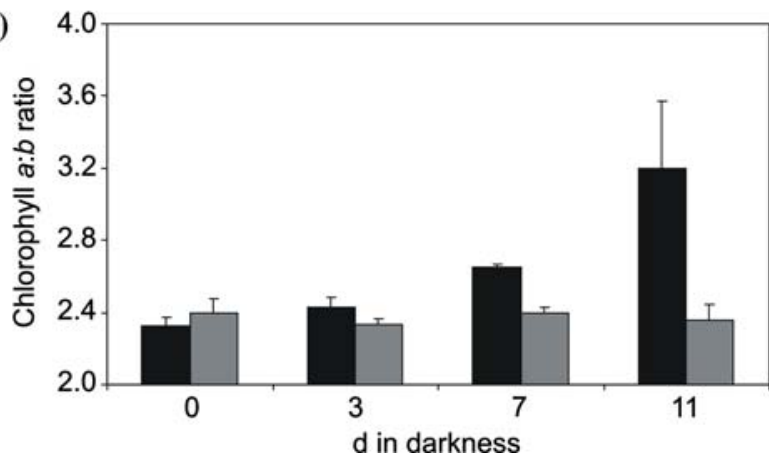

Fig. 1 Phenotype of the green cotyledon mutant J12775 during chl breakdown. (a) Phenotype of individual leaves of JI4 (left) and JI2775 (right) after 11 days of dark-induced (upper panel) or natural senescence (lower panel). Inset, mature seeds from JI4 (left) and JI2775 (right) with the testa removed. Scale bars, $1 \mathrm{~cm}$. (b) Degradation of chl in JI4 (black) and JI2775 (gray) during the course of dark-induced senescence. Data are means of a single representative experiment with three replicates. Error bars indicate SD. (c) Chl $a: b$ ratio in JI4 (black) and JI2775 (gray) during the course of darkinduced senescence. Data are means of a single representative experiment with three replicates. Error bars indicate SD

greens (Kingston-Smith et al. 1997; Thomas and Howarth 2000). To examine the correlation between the greenness and leaf functionality, we covered attached pea leaves with aluminum foil in order to induce senescence and determined the maximal fluorescence yield $\left(F_{\mathrm{v}} / F_{\mathrm{m}}\right)$ of photosystem II (PSII), reflecting the maximum quantum yield of the primary photochemistry of PSII (Fig. $2 \mathrm{a}) . F_{\mathrm{v}} / F_{\mathrm{m}}$ decreased during the dark incubation in both lines, but the decrease was somewhat slower in JI2775 compared to JI4. We further analyzed $F_{0}$, an indicator of the connectivity of PSII 
antenna with the core center (Havaux 1993; Oh et al. 2000). During leaf senescence, $F_{0}$ increased more drastically in JI4 (Fig. 2b), indicating dissociation of LHCII complexes from the PSII core. In contrast, in the mutant PSII integrity seemed to be retained much longer. Finally, the quantum yield of PSII electron transport $\left(\Phi_{\text {PSII }}\right)$ decreased slower in JI2775, but after 11 days had reached similar values like JI4 (Supplemental Fig. 1). Together, these fluorescence parameters indicated that senescence-related reduction of photosynthetic activity was somewhat slower in J12775, but eventually reached levels comparable to the wild type. Moreover, the rate of net photosynthesis as determined by measuring net $\mathrm{CO}_{2}$ fixation, decreased similarly during the course of senescence in both lines (Fig. 2c). Hence, photosynthetic activity was uncoupled from chl metabolism in the mutant, qualifying JI2775 a type C stay-green mutant according to a recently suggested nomenclature of staygreen mutants (Thomas and Howarth 2000).

To further characterize the senescence behavior of JI2775, expression of SAG12, a commonly accepted marker of senescence (Quirino et al. 2000), was investigated by semiquantitative RT-PCR. In both lines, expression of PSSAG12 increased to a similar extend during darkinduced senescence (Fig. 2d), indicating that senescence was initiated normally in the mutant.

We analyzed protein metabolism as a further marker of senescence progression. Again JI2775 behaved like JI4, i.e. overall protein degradation during dark-induced senescence was rather similar in both lines (data not shown). Nevertheless, differences were observed when individual photosynthesis-related proteins were analyzed by immunoblotting (Fig. 3a, b). LHCII was fully retained during the course of senescence in J12775, a feature that has been described in different stay-green mutants (Hilditch et al. 1989; Bachmann et al. 1994; Pružinská et al. 2003; Park et al. 2007), but in contrast to the stay-green mutant Bf993 of Festuca pratensis (Thomas and Howarth 2000), proteolytic fragments of LHCII could not be detected in JI2775. In addition, core subunits of PSI (PsaD) and PSII (PsbA) were also retained to some extend in the mutant, and degradation of Rubisco subunits was somewhat slower in JI2775 compared to JI4. Together these data confirm earlier observations of high LHCII retention in stay-green mutants (Hilditch et al. 1989; Jiang et al. 2007; Park et al. 2007).

Overall, the diverse physiological data presented here confirm that J12775 exhibits typical features of non-functional, type $\mathrm{C}$ stay-green mutants.

Isolation and characterization of $S G R$ genes and proteins

By genomic mapping, SGR has been shown to locate to the I locus of Gregor Mendel's green cotyledon mutant
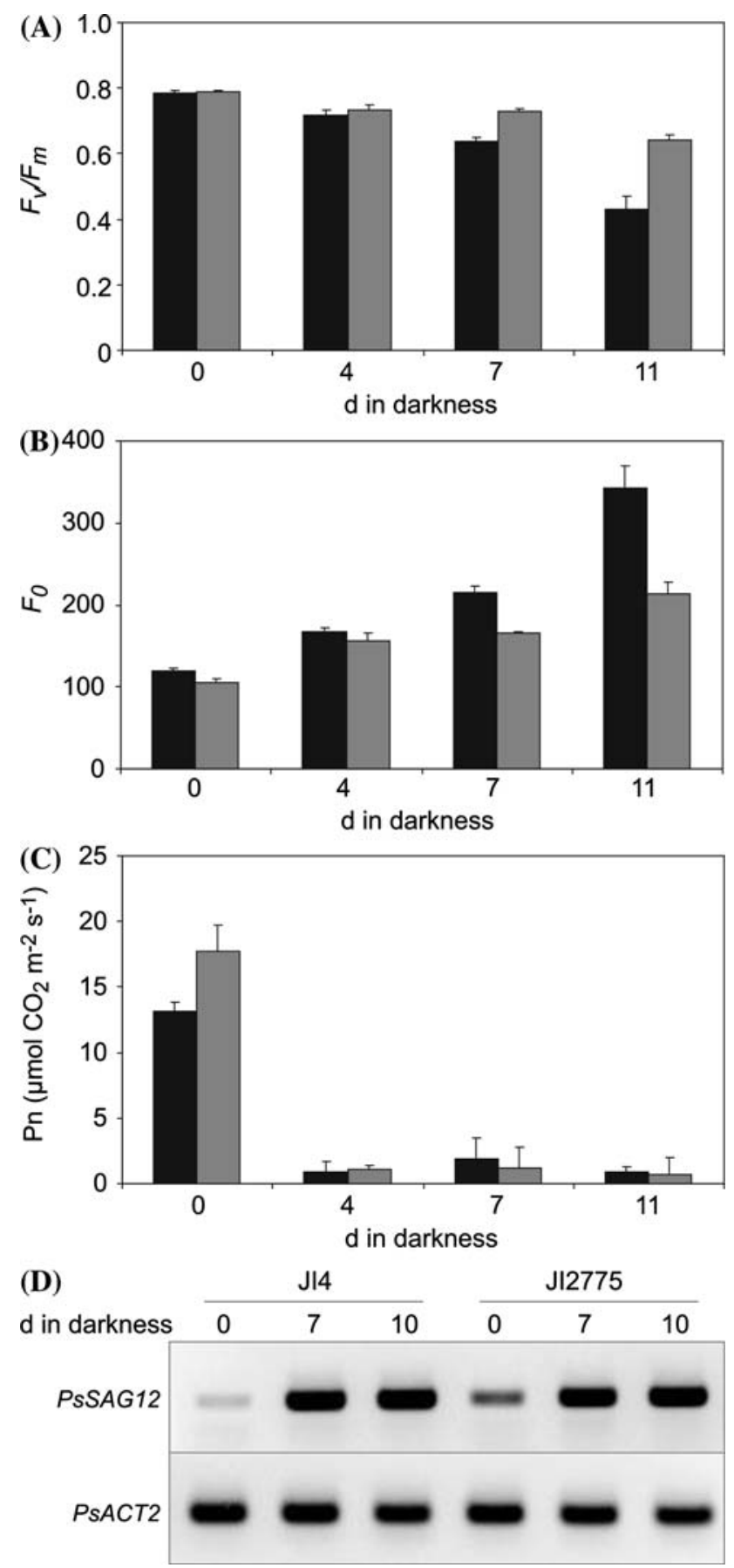

Fig. 2 Characterization of the senescence behavior of JI4 and JI2775. $(\mathbf{a}-\mathbf{c})$ Maximum quantum yield of PSII $\left(F_{\mathrm{v}} / F_{\mathrm{m}} ; \mathbf{a}\right)$, minimum fluorescence in the dark $\left(F_{0} ; \mathbf{b}\right)$ and net photosynthesis $(\mathrm{Pn} ; \mathbf{c})$ during leaf senescence in JI4 (black) and JI2775 (gray). For senescence induction, attached leaves were covered with aluminum foil. Data are means of a single representative experiment with 6 replicates. Error bars indicate SE. (d) Semiquantitative analysis of expression of PSSAG12 during senescence. PSACT2 expression was used as control

(Armstead et al. 2007). This was recently indirectly confirmed by a segregation analysis exploiting the size differences of intron three of the pea $S G R$ gene (Sato et al. 

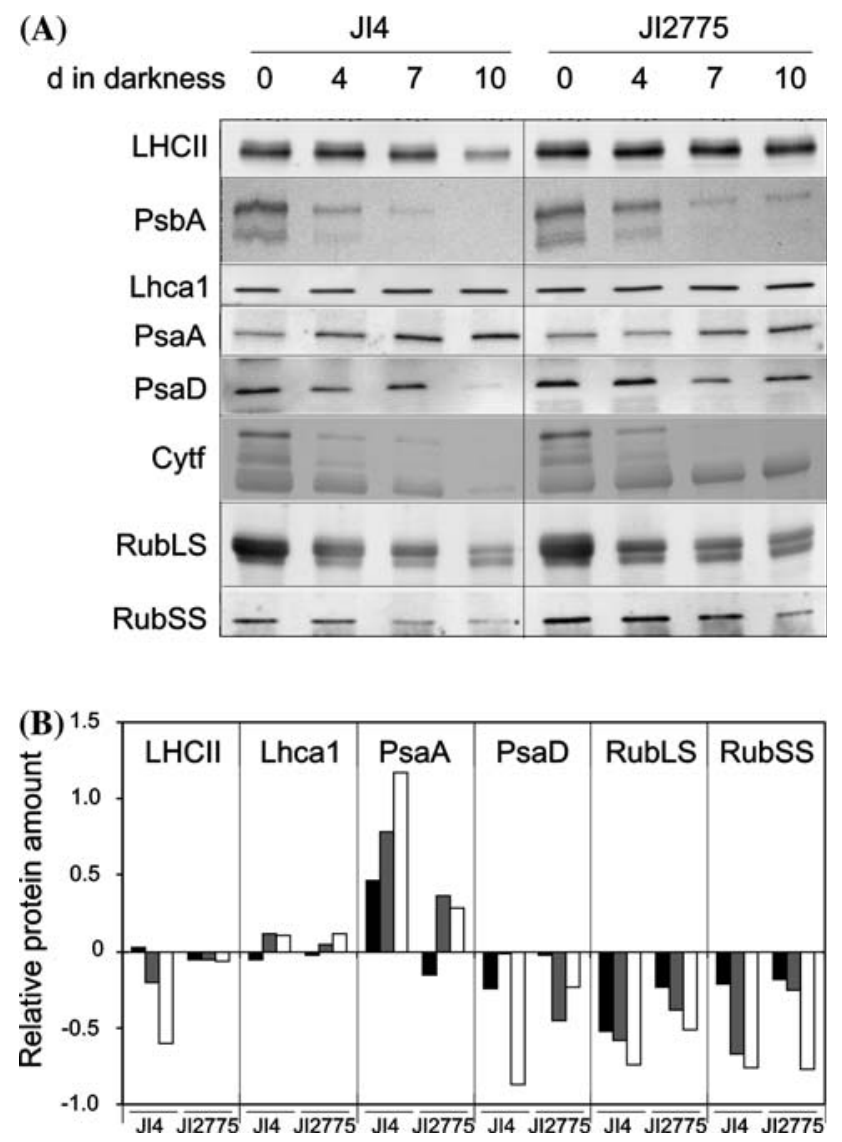

Fig. 3 Analysis of protein degradation during leaf senescence of JI4 and J12775. (a) Immunoblot analysis of photosynthesis-related protein abundance in leaves of JI4 and JI2775 during dark-induced senescence. Antibodies against the following proteins were employed: LHCII and PsbA (photosystem II), LhcaI, PsaA and PsaD (photosystem I), Cytf (cytochrom $\mathrm{b}_{6} \mathrm{f}$ complex) and Rubisco large (RubLS) and small subunit (RubSS). Gel loadings are based on equal amounts of fresh weight. (b) Relative changes of protein abundance as determined by quantifying the chemiluminescence signals of blots shown in (a). Bars represent relative changes of protein amounts after 4 days (black), 7 days (gray) and 10 days (white) of senescence as compared to 0 days samples. Thereby, negative values reflect degradation; positive values reflect increase of respective protein amounts. PsbA and Cytf blots (panel a) were visualized with a color substrate and, therefore, signals were not quantified

2007). We cloned the coding region of PSSGR from genomic DNA isolated from JI4 and JI2775. Thereby the genomic sequence was $81 \%$ and the coding sequence $99.8 \%$ identical between the two lines. Sequence differences were found at the same three positions of the coding region as described before (Sato et al. 2007), resulting in two missense mutations (Thr12Ser and Asn38Lys) and an Ile-Leu insertion after Ile189 of the deduced SGR protein of JI2775 as compared to JI4. Surprisingly, within the introns, sequence differences were limited to the third intron with an increase in size from 802 bases (JI4 SGR gene) to 923 bases in JI2775. Sequence identity of the third intron was $64 \%$.
The completed Arabidopsis and rice genomes as well as additional available genomic sequences of SGRs were used to compare the exon/intron structure of SGR genes (Fig. 4a). The exact positions of introns were deduced by alignment of the genomic regions with expressed sequence tag (EST) sequences from respective species. The gene structure was conserved within the monocots and dicots, but differed from each other by the presence in the dicot genes of an additional intron splitting exon 2 of the monocot SGR genes (Fig. 3a). In all cases, introns were present at exactly the same positions within the coding regions indicating that SGR genes had existed prior to monocot and dicot divergence about 200 million years ago and that divergence is characterized by the occurrence of the additional intron. Consequently SGR-like genes are also found in lower plants, such as Gymnosperms (Ren et al. 2007), Physcomitrella patens, and eukaryotic algae, but also in some bacteria. The derived proteins cluster into several subclades, whereby monocot (clade I) and dicot SGR members (clade II) cluster together and are separated from lower species (Supplemental Fig. 2). Interestingly, a phylogenetically distant subclade of SGR homologues (clade III) contained further proteins from different higher plants, such as Arabidopsis and rice. In most cases of higher plant SGRs, expression of respective genes is supported by the presence of ESTs (not shown), but it remains to be demonstrated whether lower plant SGRs are functional orthologs of the higher plant proteins. An interesting feature of the monocot and dicot sequences in clade I and II, and of two $P$. patens sequences was the presence of a novel conserved motif near the C-terminal ends (Fig. 4b). The motif can be defined as Cys- $\mathrm{X}_{3}$-Cys-X-Cys-Cys-PhePro- $\mathrm{X}_{7}$-Pro and is separated from the highly homologous core region of SGRs by a variable region of 12-38 amino acids in length. This motif was absent from the SGR-like proteins of clade III, and from lower plant and bacterial homologues. The presence of 4 conserved Cys residues implies possible functions in inter- or intramolecular crosslinking, or in redox regulation, but this has not yet been experimentally tested.

Interestingly, different isoforms are found in the (nearly) finished sequenced genomes of several species, such as Arabidopsis thaliana, Vitis vinifera, Populus trichocarpa, maize and rice. In Arabidopsis three SGR genes have been annotated, but a fourth gene, AtSGR4, is present as a tandem repeat of AtSGR2 (At4g11910). This most likely represents a non-functional pseudogene, since ESTs cannot be identified in the available databases (data not shown). In order to identify the $S G R$ gene number in pea, Southern blot analysis was performed with restricted genomic DNA of JI4 and JI2775 (Fig. 4c). The pattern of bands hybridizing with a $P S S G R$ cDNA probe, indicate the presence in both pea lines of two $S G R$ genes. This is confirmed by the 


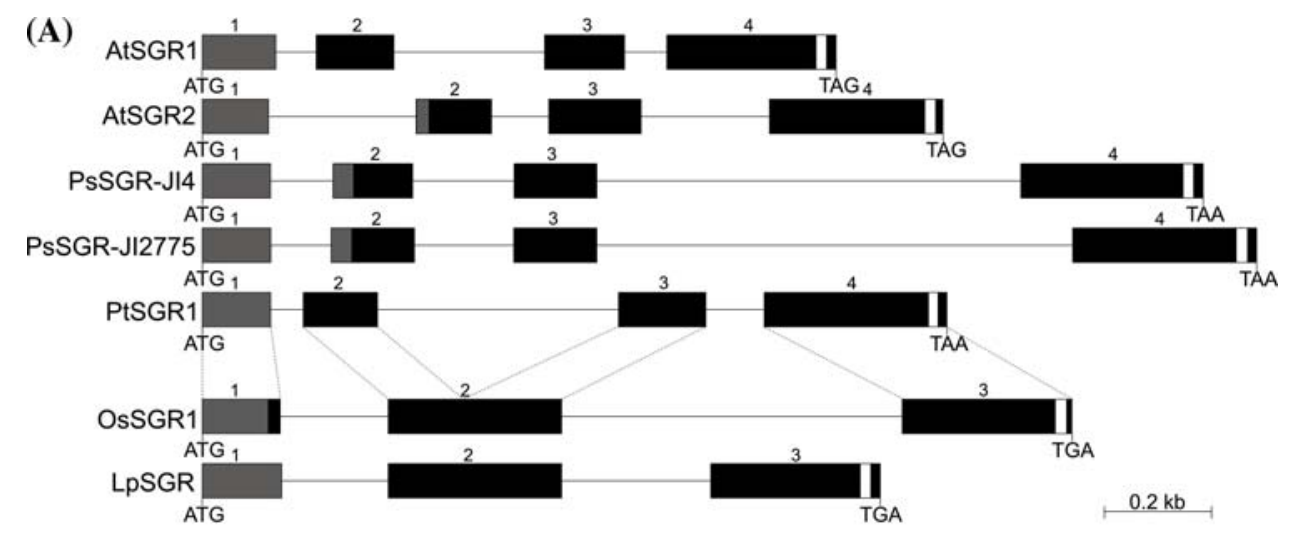

(B)

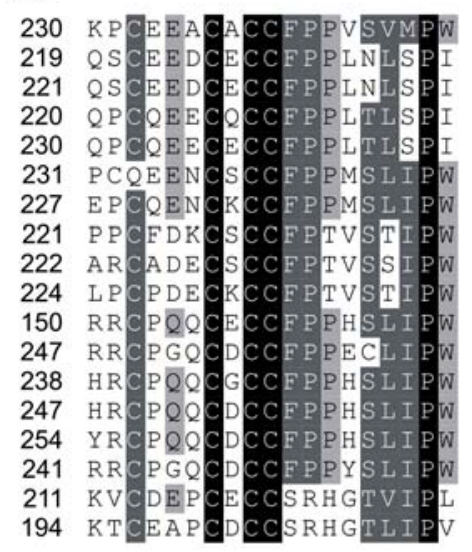

LeSGR

PsSGR-JI4

PsSGR-JI2775

GmSGR1

GmSGR2

PtSGR1

PtSGR2

AtSGR4

AtSGR1

AtSGR2

ZjSGR

HvSGR

ZmSGR2

ZmSGR1

SbSGR

OsSGR1

PpSGR1

PpSGR2

(D)

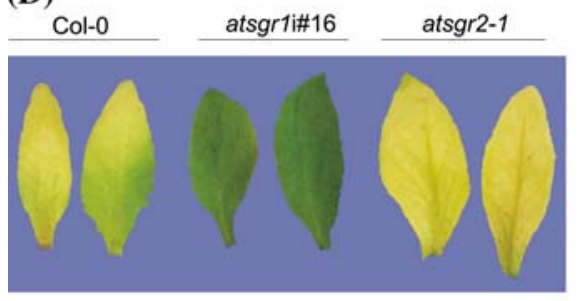

(C)

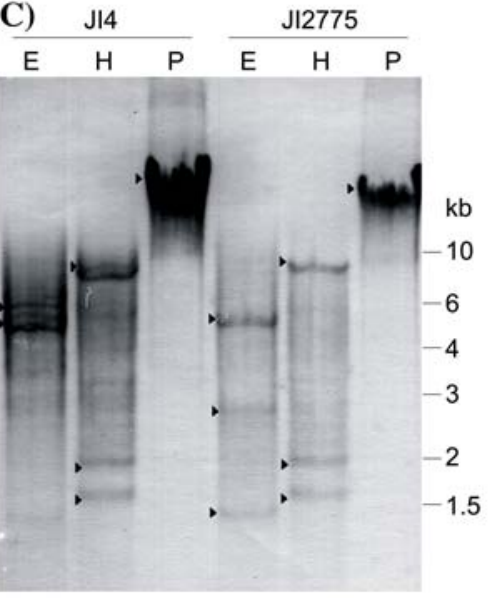

(E)

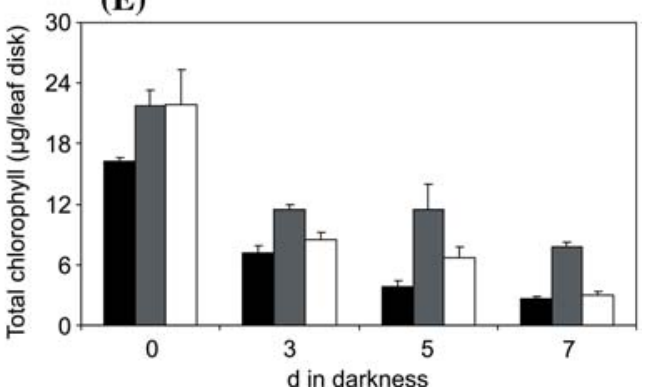

as $\mathrm{C}-\mathrm{X}_{3}-\mathrm{C}-\mathrm{X}-\mathrm{C}_{2} \mathrm{FP}-\mathrm{X}_{7}-\mathrm{P}$. Amino acid sequences were aligned using Dialign (http://bibiserv.techfak.uni-bielefeld.de/dialign). Black shading with white letters, gray shading with white letters, and gray shading with black letters reflect 100 , 80 , and $60 \%$ sequence conservation, respectively, with Blosum62 similarity groups enabled. For accession numbers of protein sequences, see Experimental procedures. (c) DNA gel blot analysis of PsSGR. $15 \mu \mathrm{g}$ of genomic DNA from both JI4 and JI2775 were digested by EcoRI (E), HindIII (H) or $P s t \mathrm{I}(\mathrm{P})$ and probed with a digoxygenin-labeled PsSGR fragment from JI4. Arrowheads indicate cross-reacting bands. Sizes of DNA marker bands are indicated on the right. (d) Phenotype of leaves of Col-0, a SGR1 silencing line (Armstead et al. 2007; atsgrli\#16) and a T-DNA insertion line of AtSGR2 (atsgr2-1) after 5 days of dark-induced senescence. (e) Degradation of chl in Col-0 (black), atsgrli\#16 (gray) and atsgr2-1 (white) during the course of detached leaf senescence. Data are means of a single representative experiment with 3 replicates. Error bars indicate SD. 
Fig. 5 PsSGR mRNA and protein abundance, and PsSGR protein functionality test. (a) Semiquantitative analysis of $P S S G R$ expression during leaf senescence in JI4 and JI2775. RNA abundance of PsACT2 was used as control (b) Real-time RT-PCR quantification of PsSGR expression during senescence. Levels of expression were normalized to levels of PSACT2 mRNA. Values are means of three replicates. (c) Immunoblot analysis of PsSGR protein levels during the course of detached leaf senescence. Gel loadings are based on equal amounts of fresh weight. (d) Transient expression of PSSGR in 3-week-old N. benthamiana plants. Leaves were infiltrated with Agrobacterium tumefaciens cultures containing plasmids for expression of PsSGRJI2775 (JI2775), PsSGR-JI4 (JI4), or empty vector (Mock). Pictures show plants after 6 days of infiltration. (e, f) Analysis of leaf tissue after infiltration as in (d). (e) Total chl levels; (f) Immunoblot analysis of LHCII, PAO and SGR levels in the treatments. Gel loadings are based on equal sizes of leaf material. Control, non-infiltrated leaf tissue

presence in the databases of a pea EST (GenBank accession number AM162161), which is highly homologous, but not identical to PSSGR.

Two functional SGR genes have been identified in Arabidopsis, but so far only for the senescence-regulated AtSGRl (At4g22920) has an important function in chl breakdown been demonstrated (Armstead et al. 2007; Ren et al. 2007). In order to analyze whether AtSGR2, whose expression is also up-regulated during senescence (Park et al. 2007), is involved in chl catabolism as well, a homozygous T-DNA insertion line (SALK_003830C) was obtained from the SALK resource (Alonso et al. 2003). The line was termed atsgr2-1 and position of the T-DNA within the first intron was confirmed by sequencing (Supplemental Fig. 3A). Expression of AtSGR2 was absent (Supplemental Fig. 3B) indicating that atsgr2-1 represents a null allele. During dark-induced senescence, chl was degraded at similar rates like in Col-0, in contrast to an AtSGR RNA silencing line (atsgrli\#16) (Armstead et al. 2007), which retained a large proportion of chl after 7 days of senescence (Fig. 4d, e). Although in atsgrli\#16, expression of AtSGR2 was also slightly reduced (Supplemental Fig. 3B), silencing of AtSGRI expression in the knockout line atsgr2-1 did not enhance the stay-green phenotype observed in atsgrli (data not shown). This indicated that AtSGR2 does not significantly contribute to chl degradation in Arabidopsis. Likewise, the presence of two or more SGR gene copies in different species indicates either redundancy of SGR function in chl breakdown or implies that SGR might have additional role(s) in plant development that so far have escaped detection. Furthermore, the unclear role of the structurally similar SGR isoproteins of higher plants, such as AtSGR2, even more questions a function in chl breakdown of the less homologous SGR-like proteins from higher (clade III proteins) and lower plants (Supplemental Fig. 2). This has to be functionally tested, e.g. through complementation of the nye1 mutant of Arabidopsis.
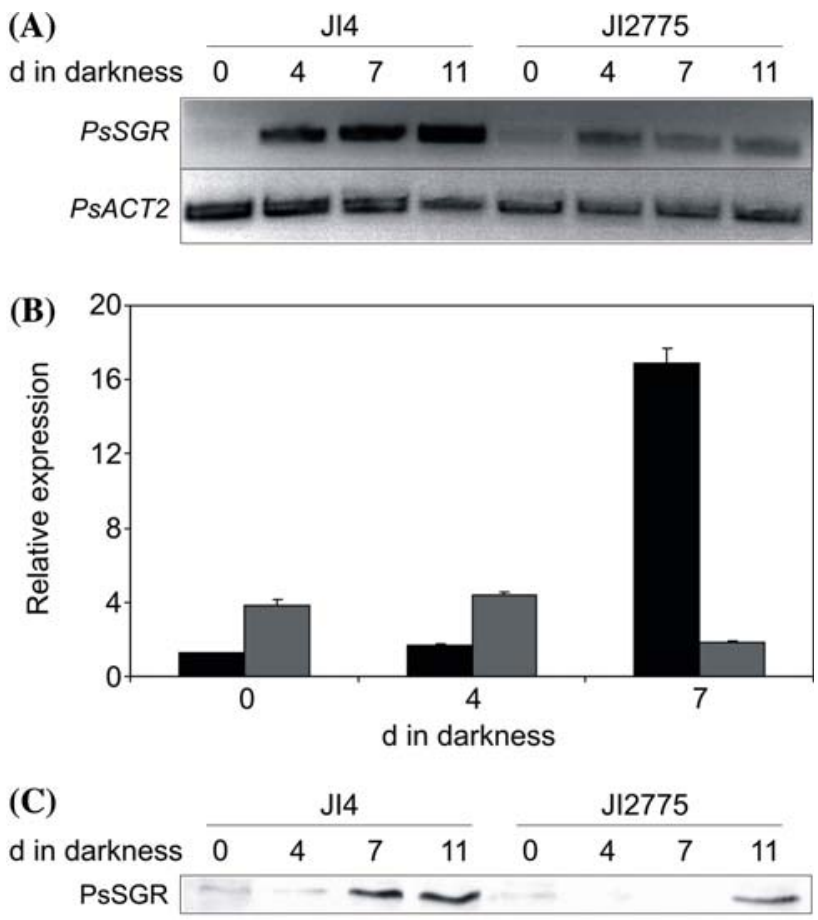

(D)
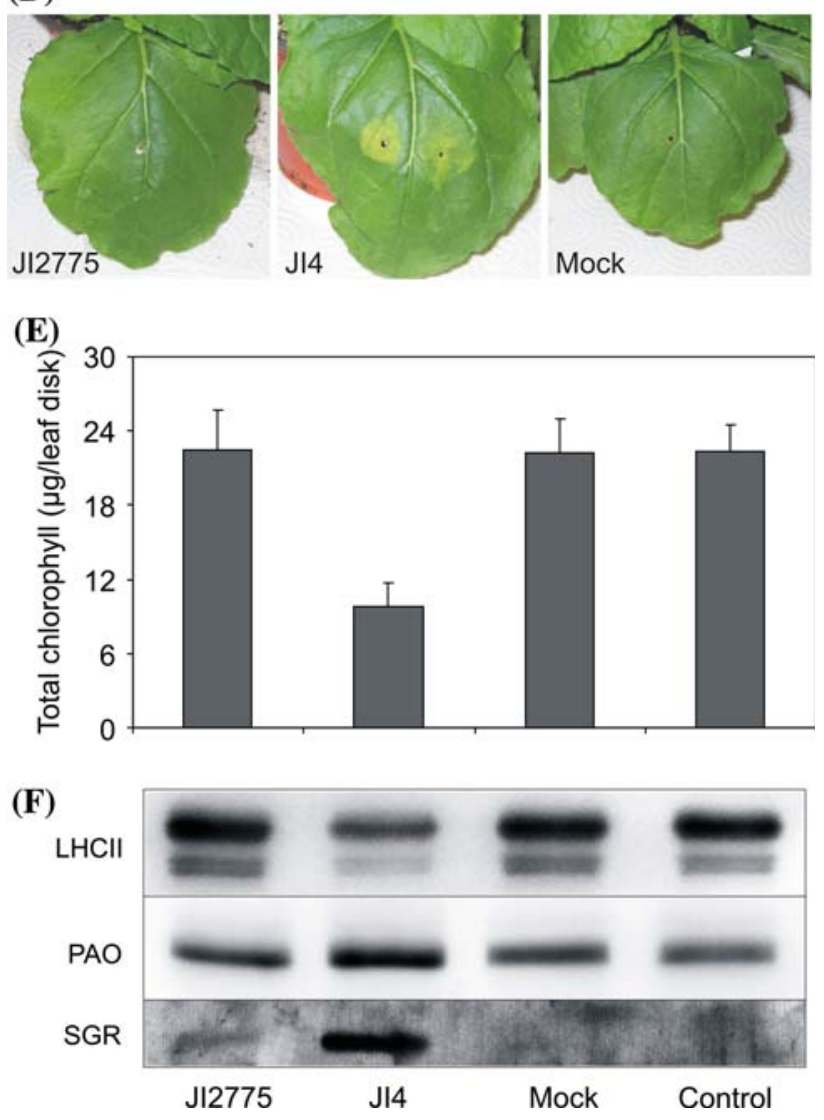

JI2775 is a loss-of-function mutant

In order to determine the molecular or biochemical defect of the pea mutant J12775, we analyzed PsSGR gene 
Fig. 6 Absence of SGR does not affect PAO abundance and activity. (a) Immunoblot analysis of PAO levels during the course of detached leaf senescence in JI4 and JI2775. Gel loadings are based on equal amounts of fresh weight. (b) Formation of pFCC-2 in PAO/RCCR assays with PAO extracted from leaves of JI4 or JI2775 after 8 days of dark-induced senescence. Activities are based on equal amounts of fresh weight. HPLC traces (fluorescence at 320/450 nm) are shown. Inset, Immunoblot analysis of PAO abundance in the extracts used for PAO/RCCR assays. Gel loadings are based on equal amounts of fresh weight. (c) Immunoblot analysis of PAO levels of individual AtSGR1silencing lines (atsgrli; Armstead et al. 2007) after 5 days of darkinduced senescence. Gel loadings are based on equal amounts of fresh weight. (d) HPLC traces of leaf extracts from pea JI4 and JI2775, and of a Lolium stay-green line (Lolium SG; Roca et al. 2004) after 8 and 5 days of dark-induced senescence, respectively, in the absence or

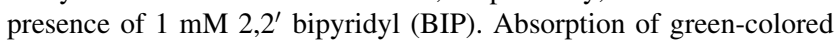
chl catabolites was monitored at $665 \mathrm{~nm}$. Analyzed extracts correspond to equal amounts of fresh weight. Pigments were identified by their absorption spectra and by comparison to authentic standards. Pheide, standard containing both, pheide $a$ and pheide $b$. The asterisk marks a non-identified peak exhibiting a chl $a$ spectrum

expression during leaf senescence by semiquantitative (Fig. 5a) and real time RT-PCR (Fig. 5b). As shown earlier for rice and Arabidopsis SGRs (Jiang et al. 2007; Park et al. 2007; Ren et al. 2007), PsSGR expression was strongly induced upon senescence induction of JI4. In contrast, mRNA levels of JI2775 were kept on a lower level during the course of senescence. This result was obtained repeatedly in independent samples and confirmed the low signals obtained in Northern blots from senescent JI2775 leaves (Armstead et al. 2007). Sato et al. (2007) reported that $P S S G R$ gene expression was unaltered in JI2775. To further analyze this discrepancy, SGR protein levels were analyzed by immunoblotting using an antiserum directed against conserved motifs of SGR. In line with our expression analysis, the senescence-related increase in SGR protein observed in JI4 was significantly reduced in the mutant (Fig. 5c). Together these data indicate that the defect in JI2775 likely results from reduced expression of PSSGR.

Nevertheless, the deduced protein sequence of SGR from JI2775 exhibited changes compared to JI4-SGR, in particular a two-amino acid insertion within a highly conserved region that could affect SGR function. Therefore, binary constructs were produced for both gene versions in order to transform JI2775. Unfortunately, it was not possible to regenerate genetically transformed immature embryos from JI2775 (A. Nadolska-Orczyk, personal communication). Instead, we addressed this question by heterologous transient expression in Nicotiana benthamiana leaves of SGR cDNA from either JI4 or JI2775. Five days after infiltration, loss of chl was observed in the leaf areas of plants that had been challenged with JI4-derived SGR (Fig. 5d, e). In contrast, expression of SGR from J12775 did not induce chl breakdown in $N$. benthamiana

\begin{tabular}{|c|c|c|c|c|c|c|c|}
\hline (A) & & & & & & & \\
\hline$d$ in darkness & 4 & 7 & 11 & 0 & 4 & 7 & 11 \\
\hline PAO & - & - & - & . & $=$ & -1 & $\rightarrow$ \\
\hline
\end{tabular}

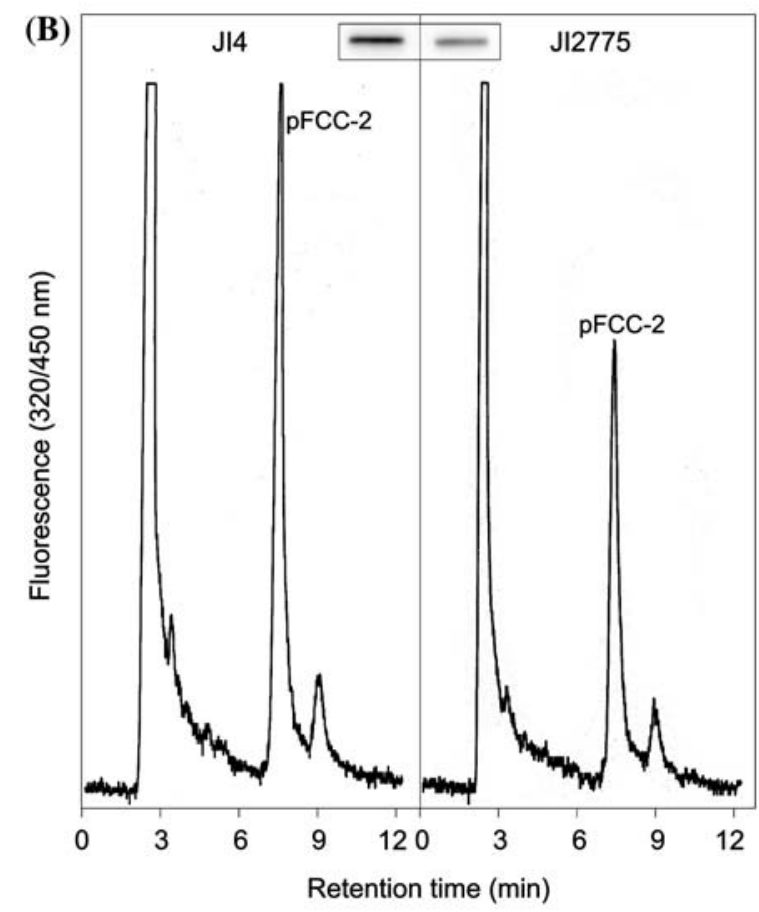

(C)

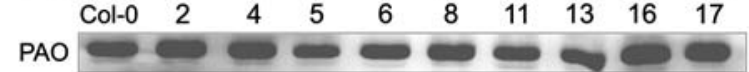

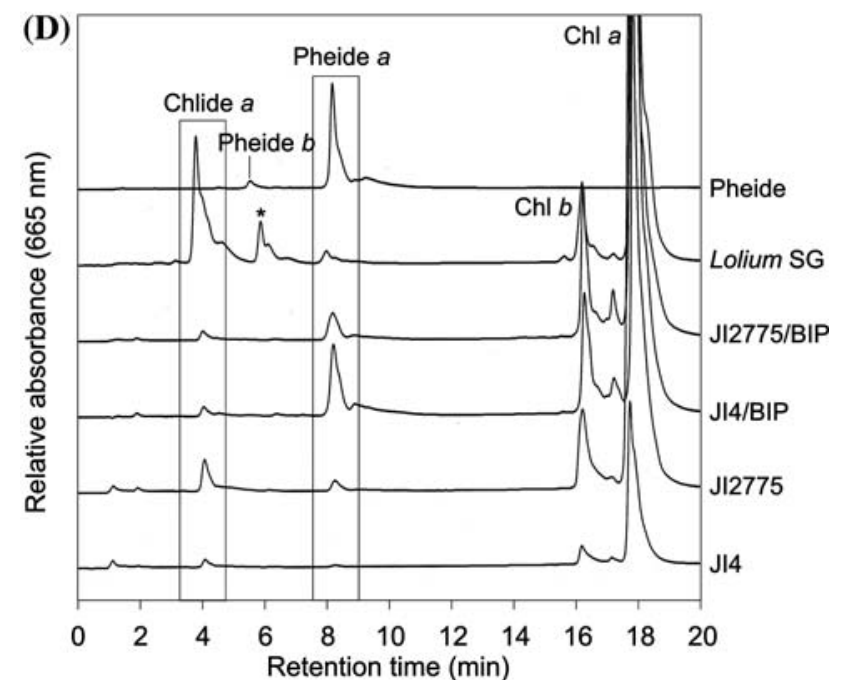

(Fig. 5d, e). Induction of senescence in the case of JI4-SGR expression was confirmed by immunoblot analyses of total protein extracts, which showed increased levels of PAO as well as degradation of LHCII proteins (Fig. 5f). Although $S G R$ expression was comparable in both cases (data not shown), much less protein accumulated in the case of JI2775-SGR, compared to JI-SGR (Fig. 5f). This directly 
demonstrated a loss of function of the mutated SGR, most likely caused by a loss of protein stability and, thus, confirms the indirect functional test performed by Sato et al. (2007). In conclusion, JI2775 is a loss-of-function mutant, which results from both reduced $S G R$ gene expression and loss of SGR protein function.

SGR absence in JI2775 does not influence the PAO pathway

It had been proposed that the stay-green phenotype of the SGR/SID-deficient mutants Bf993 of Festuca pratensis, nyel of Arabidopsis and Mendel's green cotyledon mutant was caused by the (partial) loss of PAO function, which was in line with the observed accumulation during senescence of pheide $a$ (Vicentini et al. 1995; Thomas et al. 1996; Roca et al. 2004; Ren et al. 2007). We re-addressed this assumption using JI4 and JI2775 and determining PAO abundance and activity. During 11 days of dark-induced senescence, PAO contents increased to comparable levels in both lines as judged by immunodetection (Fig. 6a). On the other hand, after extraction from senescent chloroplast membranes according to an established protocol (Hörtensteiner et al. 1995; Pružinská et al. 2005), PAO activity was $40 \%$ lower in extracts of JI2775 compared to the wild type (Fig. 6b). At first glance, this result indicated a posttranslational regulation of PAO, as has been proposed in canola seed development (Chung et al. 2006). In contrast, data on expression, protein abundance and activity suggest that PAO is transcriptionally regulated during Arabidopsis leaf senescence (Pružinská et al. 2005). When determining by immunoblotting the PAO content within the pea membrane extracts used for PAO assays, proportionally less PAO was found in the PAO extracts from the mutant (Fig. 6b, inset). This could be explained by the presence in senescent JI2775 leaves of large quantities of chloroplast membranes, which made a quantitative extraction of PAO impossible. We conclude that PAO abundance (and activity) are unchanged in the mutant compared to JI4. Likewise, PAO levels of senescent leaves were unaltered in different SGR-silencing lines of Arabidopsis (Fig. 6c). The same was true for a Lolium introgression line carrying the stay-green allele of Festuca pratensis Bf993 (H. Ougham, S. Hörtensteiner, I. Armstead, I. Donnison, I. King, H. Thomas and L. Mur, unpublished), although there lower PAO activities had been observed as well (Roca et al. 2004). Finally, Ren et al. (2007) also identified lower PAO activity in nye1, but unfortunately PAO protein abundance was not determined in this study. Altogether, the observed lower PAO activities in sgr/sid mutants (Fig. 6b; Vicentini et al. 1995; Roca et al. 2004; Ren et al. 2007) are due to misinterpretation of results and no indication remains of a posttranslational regulation of PAO through SGR as suggested (Sato et al. 2007).

We also re-investigated the observation of pheide $a$ accumulation in $s g r / s i d$ mutants. Pheide $a$ did not accumulate in senescent JI4 leaves, but small quantities (5-10 nmol pheide $a \mathrm{~g}^{-1}$ fresh weight) were found in JI2775 (Fig. 6d). If the occurrence in JI4 of pheide $a$ would solely be the result of partial inhibition of PAO in an otherwise normally active chl breakdown pathway, it could be expected that entire inhibition of PAO would increase the amount of pheide $a$. To analyze this, leaves were senesced in the presence of 2,2'-bipyridyl, an iron chelator, which among other reactions has been shown to inhibit PAO (Hörtensteiner et al. 1995; Hörtensteiner et al. 2000). In wild type, bipyridyl-treatment largely enhanced the accumulation of pheide $a$ ( $80 \mathrm{nmol} \mathrm{g}^{-1}$ fresh weight), but this increase was much smaller in JI2775 $\left(35 \mathrm{nmol} \mathrm{g}^{-1}\right.$ fresh weight). These data indicate that occurrence of pheide $a$ is independent of PAO. Instead, pheide $a$ formation could result from non-physiological conversion from chlide $a$. This might be due to tissue acidification as a consequence of tonoplast rupture upon cell damage or death occurring at later stages of leaf senescence. Chlide $a$ repeatedly accumulated to higher concentrations in senescent leaves of the mutant compared to JI4 (Fig. 6d), an observation that had been made also in other instances of sgr/sid mutants (Vicentini et al. 1995; Roca et al. 2004). Particularly in Bf993 and the stay-green introgression lines of Lolium, large quantities accumulated in senescent leaves. This intriguing observation correlates to the fact that, compared to rice and pea sgr mutants, the Festuca/Lolium sid lines are null mutants, caused by a frame shift mutation within the second exon of the gene (Armstead et al. 2006). In this respect it is possible to argue that SGR/SID might affect Mg-dechelation. This step in chl breakdown was shown to be catalyzed nonenzymatically by a heat-stable, low molecular weight compound, termed metal chelating substance. Metalchelating substances of different species have different molecular weights, but their structures have so far not been elucidated (Costa et al. 2002; Suzuki et al. 2005). Alternatively, metal-chelating substance might represent the metal-chelating cofactor of a Mg-dechelatase enzyme (Hörtensteiner 2006) and it remains to be investigated whether SGR could have this function.

Altogether the data presented here refute the suggestion of several groups of impairment of PAO function in SGR mutants, hence, most likely the biochemical steps of the PAO pathway of chl breakdown are not regulated by SGR.

\section{SGR acts upstream of PAO}

If SGR does not regulate the biochemical enzymes of chl breakdown, at which level does it then interfere with chl 


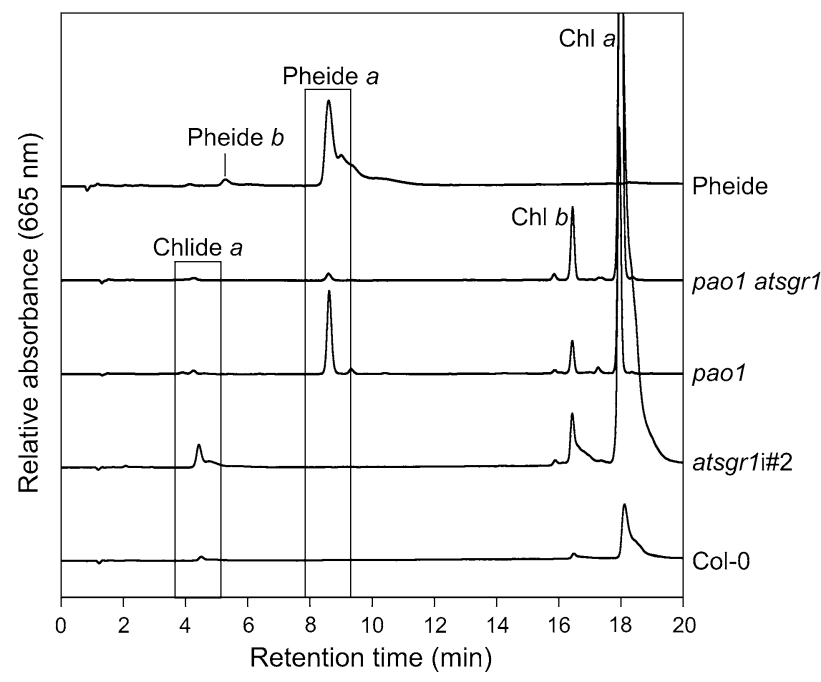

Fig. 7 HPLC analysis of green chl catabolites in SGRI- and PAOdeficient Arabidopsis. Equal amounts of leaf extracts from Col-0, atsgrli\#2 (Armstead et al. 2007), paol (Pružinská et al. 2005) and a representative line silencing AtSGR1 in paol background (paol atsgrli) were separated by reversed-phase HPLC, and $A_{665}$ was monitored. Pigments were identified by their absorption spectra and by comparison to authentic standards. Pheide, standard containing both, pheide $a$ and pheide $b$

metabolism? In order to address this question, we silenced expression of AtSGRl in the paol background (Pružinská et al. 2005). Two independent homozygous lines, paol atsgrli\#1 and paol atsgrli\#2, were isolated and further analyzed. During senescence, the lines exhibited a staygreen phenotype, but a cell death phenotype, typical for paol, did not establish in the paol atsgrli lines (data not shown). Furthermore, in contrast to paol, pheide $a$ accumulation was largely reduced in the paol atsgrli lines (Fig. 7). This result indicated that abolishment of SGR function prevents pheide $a$ formation, hence confirms that the SGR target is located upstream of PAO.

\section{Conclusion}

The identification and analysis of the role of SGR/SID proteins during plant senescence has recently become a major focus in plant senescence research. This is demonstrated by the high number of reports about identification of sgr/sid mutants and the recent cloning of SGR/SID genes from Arabidopsis, Festuca/Lolium, pea and rice (Armstead et al. 2006, 2007; Jiang et al. 2007; Park et al. 2007; Ren et al. 2007; Sato et al. 2007). It is likely that additional stay-green mutants, such as tomato green flesh (gf; Akhtar et al. 1999) and pepper chlorophyll retainer ( $\mathrm{cl}$; Efrati et al. 2005) are defective in $S G R$ as well. Only some of these mutants have been attributed to the class of type $\mathrm{C}$ non-functional stay-greens. Here we show that Gregor Mendel's I mutant also belongs to this class.

Analysis of the different $\mathrm{sgr} / \mathrm{sid}$ mutants available so far, indicate that overall reduction of SGR gene expression (Armstead et al. 2007) or loss of function due to mutations within the open reading frames of SGR genes (Jiang et al. 2007; Park et al. 2007; Ren et al. 2007) can cause the staygreen phenotype. In this respect, Mendel's $I$ mutant is exceptional, since we here show that both gene expression and protein function are affected. An explanation for the reduced mRNA level most probably is an altered SGR promoter activity in the mutants, but this remains to be confirmed.

SGR/SID proteins constitute a new class of senescence proteins, which are widely distributed in plants. The absence of known domains makes it impossible to deduce a function from the primary sequence of SGR proteins. Conflicting reports in the literature indicated an activity of SGR in relation to either PAO function and/or chl-apoprotein dismantling (Vicentini et al. 1995; Thomas et al. 1996; Roca et al. 2004; Park et al. 2007; Ren et al. 2007). With the results presented here, we unambiguously demonstrate that SGR does not regulate PAO. We furthermore show that SGR functions upstream of PAO, but the exact site of action remains to be elucidated. Park et al. (2007) showed physical interaction between SGR and LHCII subunits and suggested that SGR might be required for dismantling of chl-apoprotein complexes. In this case SGR would senso stricto not be part of the catabolic machinery of chl breakdown directly, but its action would be a prerequisite for allowing the catabolic enzymes to get access to their substrate. An interesting observation is that in paol $S G R$ gene expression is not up-regulated during senescence (Park et al. 2007). It is likely that this regulation is achieved through pheide $a$, although, in contrast to the suggested retrograde signaling by porphyrin intermediates of chl biosynthesis (Nott et al. 2006), pheide $a$ most probably is not a retrograde signal itself. Nevertheless, the proposed function in the dismantling of chlprotein complexes (Park et al. 2007) provides an ideal point of control of chl breakdown. In addition, the retention of chl-binding proteins suggests that proteases known to degrade LHC proteins during senescence, such as FtsH6 (Zelisko et al. 2005), are either directly regulated through SGR or complex-dismantling is also a prerequisite for the respective proteases to get access to their substrate proteins. In this respect, the analysis of the rice nycl mutant provides an interesting alternative (Kusaba et al. 2007); chl $b$ is known to be required in a certain stoichiometry to chl $a$ in order to stabilize chl-protein complexes (Horn and Paulsen 2004), thus, prevention of chl $b$ to chl $a$ conversion in nycl would stabilize the complexes. It will be interesting to analyze the regulation of SGR in $n y c l$ and to 
see whether NYC1 or SGR or both regulate chl-protein complex stability.

Acknowledgements We thank B. Burla, University of Zurich, for his help with phylogenetic analyses. We thank S. Schelbert, University of Zurich, for critical reading of the manuscript and I. Anders, University of Bern, for technical support. This work was supported by the Swiss National Science Foundation (3100A0-105389) and the National Center of Competence in Research Plant Survival, research program of the Swiss National Science Foundation.

\section{References}

Akhtar MS, Goldschmidt EE, John I, Rodoni S, Matile P, Grierson D (1999) Altered patterns of senescence and ripening in $g f$, a staygreen mutant of tomato (Lycopersicon esculentum Mill.). J Exp Bot 50:1115-1122

Alonso JM, Stepanova AN, Leisse TJ, Kim CJ, Chen H, Shinn P, Stevenson DK, Zimmermann J, Barajas P, Cheuk R, Gadrinab C, Heller C, Jeske A, Koesema E, Meyers CC, Parker H, Prednis L, Ansari Y, Chory N, Deen H, Geralt M, Hazari N, Hom E, Karnes M, Mulholland C, Ndubaku R, Schmidt I, Guzman P, Aguilar-Henonin L, Schmid M, Weigel D, Carter DE, Marchand T, Risseeuw E, Brogden D, Zeko A, Crosby WL, Berry CC, Ecker JR (2003) Genomewide insertional mutagenesis of Arabidopsis thaliana. Science 301:653-657

Armstead I, Donnison I, Aubry S, Harper J, Hörtensteiner S, James C, Mani J, Moffet M, Ougham H, Roberts L, Thomas A, Weeden N, Thomas H, King I (2006) From crop to model to crop: identifying the genetic basis of the staygreen mutation in the Lolium/Festuca forage and amenity grasses. New Phytol 172:592-597

Armstead I, Donnison I, Aubry S, Harper J, Hörtensteiner S, James C, Mani J, Moffet M, Ougham H, Roberts L, Thomas A, Weeden N, Thomas H, King I (2007) Cross-species identification of Mendel's $I$ locus. Science 315:73

Bachmann A, Fernández-López J, Ginsburg S, Thomas H, Bouwcamp JC, Solomos T, Matile P (1994) Stay-green genotypes of Phaseolus vulgaris L.: chloroplast proteins and chlorophyll catabolites during foliar senescence. New Phytol 126:593-600

Bhattacharyya MK, Smith AM, Ellis THN, Hedley C, Martin C (1990) The wrinkled-seed character of pea described by Mendel is caused by a transposon-like insertion in a gene encoding starch-branching enzyme. Cell 60:115-122

Chakraborti D, Sarkar A, Gupta S, Das S (2006) Small and large scale genomic DNA isolation protocol for chickpea (Cicer arietinum L.), suitable for molecular marker and transgenic analyses. Afr J Biotechnol 5:585-589

Chung DW, Pružinská A, Hörtensteiner S, Ort DR (2006) The role of pheophorbide $a$ oxygenase expression and activity in the canola green seed problem. Plant Physiol 142:88-97

Costa ML, Civello PM, Chaves AR, Martinez GA (2002) Characterization of Mg-dechelatase activity obtained from Fragaria $\times$ ananassa fruit. Plant Physiol Biochem 40:111-118

Efrati A, Eyal Y, Paran I (2005) Molecular mapping of the chlorophyll retainer $(c l)$ mutation in pepper (Capsicum spp.) and screening for candidate genes using tomato ESTs homologous to structural genes of the chlorophyll catabolism pathway. Genome 48:347-351

Gray J, Wardzala E, Yang M, Reinbothe S, Haller S, Pauli F (2004) A small family of LLS1-related non-heme oxygenases in plants with an origin amongst oxygenic photosynthesizers. Plant Mol Biol 54:39-54
Havaux M (1993) Characterization of thermal damage to the photosynthetic electron transport system in potato leaves. Plant Sci 94:19-33

Hellens R, Edwards EA, Leyland NR, Bean S, Mullineaux PM (2000) pGreen: a versatile and flexible binary Ti vector for Agrobacterium-mediated plant transformation. Plant Mol Biol 42:819-832

Hilditch P, Thomas H, Rogers L (1986) Leaf senescence in a nonyellowing mutant of Festuca pratensis: Photosynthesis and photosynthetic electron transport. Planta 167:146-151

Hilditch PI, Thomas H, Thomas BJ, Rogers LJ (1989) Leaf senescence in a non-yellowing mutant of Festuca pratensis: proteins of photosystem II. Planta 177:265-272

Horn R, Paulsen H (2004) Early steps in the assembly of lightharvesting chlorophyll $a / b$ complex - Time-resolved fluorescence measurements. J Biol Chem 279:44400-44406

Hörtensteiner S (2006) Chlorophyll degradation during senescence. Annu Rev Plant Biol 57:55-77

Hörtensteiner S, Vicentini F, Matile P (1995) Chlorophyll breakdown in senescent cotyledons of rape, Brassica napus L.: enzymatic cleavage of phaeophorbide $a$ in vitro. New Phytol 129:237-246

Hörtensteiner S, Chinner J, Matile P, Thomas H, Donnison IS (2000) Chlorophyll breakdown in Chlorella protothecoides: characterization of degreening and cloning of degreening-related genes. Plant Mol Biol 42:439-450

Jiang H, Li M, Liang N, Yan H, Wei Y, Xu X, Liu J, Xu Z, Chen F, Wu G (2007) Molecular cloning and function analysis of the stay green gene in rice. Plant J 52:197-209

Kingston-Smith AH, Thomas H, Foyer CH (1997) Chlorophyll $a$ fluorescence, enzyme and antioxidant analyses provide evidence for the operation of alternative electron sinks during leaf senescence in a stay-green mutant of Festuca pratensis. Plant Cell Environ 20:1323-1337

Kreuz K, Tommasini R, Martinoia E (1996) Old enzymes for a new job. Herbicide detoxification in plants. Plant Physiol 111:349-353

Kürsteiner O, Dupuis I, Kuhlemeier C (2003) The pyruvate decarboxylase 1 gene of Arabidopsis is required during anoxia but not other environmental stresses. Plant Physiol 132:968-978

Kusaba M, Ito H, Morita R, Iida S, Sato Y, Fujimoto M, Kawasaki S, Tanaka R, Hirochika H, Nishimura M, Tanaka A (2007) Rice NON-YELLOW COLORING1 is involved in light-harvesting complex II and grana degradation during leaf senescence. Plant Cell 19:1362-1375

Lester DR, Ross JJ, Davies PJ, Reid JB (1997) Mendel's stem length gene $(L e)$ encodes a gibberellin $3 \beta$-hydroxylase. Plant Cell 9:1435-1443

Llave C, Kasschau KD, Carrington JC (2000) Virus-encoded suppressor of posttranscriptional gene silencing targets a maintenance step in the silencing pathway. Proc Natl Acad Sci USA 97:13401-13406

Matile P, Hörtensteiner S, Thomas H (1999) Chlorophyll degradation. Annu Rev Plant Physiol Plant Mol Biol 50:67-95

Maxwell K, Johnson GN (2000) Chlorophyll fluorescence-a practical guide. J Exp Bot 51:659-668

Mendel G (1866) Versuche über Pflanzenhybriden. Verh Naturforsch Ver Brünn 4:3-47

Müller T, Moser S, Ongania K-H, Pružinská A, Hörtensteiner S, Kräutler B (2006) A divergent path of chlorophyll breakdown in the model plant Arabidopsis thaliana. ChemBioChem 7:40-42

Murray MG, Thompson WF (1980) Rapid isolation of high molecular-weight plant DNA. Nucl Acids Res 8:4321-4325

Nott A, Jung HS, Koussevitzky S, Chory J (2006) Plastid-to-nucleus retrograde signaling. Annu Rev Plant Biol 57:739-759

Oberhuber M, Berghold J, Breuker K, Hörtensteiner S, Kräutler B (2003) Breakdown of chlorophyll: a nonenzymatic reaction accounts for the formation of the colorless "nonfluorescent" chlorophyll catabolites. Proc Natl Acad Sci USA 100:6910-6915 
Oh MH, Kim YJ, Lee CH (2000) Leaf senescence in a stay-green mutant of Arabidopsis thaliana: disassembly process of photosystem I and II during dark-incubation. J Biochem Mol Biol 33:256-262

Park S-Y, Yu J-W, Park J-S, Li J, Yoo S-C, Lee N-Y, Lee S-K, Jeong S-W, Seo HS, Koh H-J, Jeon J-S, Park Y-I, Paek N-C (2007) The senescence-induced staygreen protein regulates chlorophyll degradation. Plant Cell 19:1649-1664

Pružinská A, Anders I, Tanner G, Roca M, Hörtensteiner S (2003) Chlorophyll breakdown: pheophorbide $a$ oxygenase is a Riesketype iron-sulfur protein, encoded by the accelerated cell death 1 gene. Proc Natl Acad Sci USA 100:15259-15264

Pružinská A, Tanner G, Aubry S, Anders I, Moser S, Müller T, Ongania K-H, Kräutler B, Youn J-Y, Liljegren SJ, Hörtensteiner S (2005) Chlorophyll breakdown in senescent Arabidopsis leaves: characterization of chlorophyll catabolites and of chlorophyll catabolic enzymes involved in the degreening reaction. Plant Physiol 139:52-63

Pružinská A, Anders I, Aubry S, Schenk N, Tapernoux-Lüthi E, Müller T, Kräutler B, Hörtensteiner S (2007) In vivo participation of red chlorophyll catabolite reductase in chlorophyll breakdown. Plant Cell 19:369-387

Quirino BF, Noh YS, Himelblau E, Amasino RM (2000) Molecular aspects of leaf senescence. Trends Plant Sci 5:278-282

Ren G, An K, Liao Y, Zhou X, Cao Y, Zhao H, Ge X, Kuai B (2007) Identification of a novel chloroplast protein AtNYE1 regulating chlorophyll degradation during leaf senescence in Arabidopsis. Plant Physiol 144:1429-1441

Roca M, James J, Pružinská A, Hörtensteiner S, Thomas H, Ougham $\mathrm{H}$ (2004) Analysis of the chlorophyll catabolism pathway in leaves of an introgression senescence mutant of Lolium temulentum. Phytochemistry 65:1231-1238

Sato Y, Morita R, Nishimura M, Yamaguchi H, Kusaba M (2007) Mendel's green cotyledon gene encodes a positive regulator of the chlorophyll-degrading pathway. Proc Natl Acad Sci USA 104:14169-14174
Scheumann V, Schoch S, Rüdiger W (1999) Chlorophyll $b$ reduction during senescence of barley seedlings. Planta 209:364-370

Sidler M, Hassa P, Hasan S, Ringli C, Dudler R (1998) Involvement of an $\mathrm{ABC}$ transporter in a developmental pathway regulating hypocotyl cell elongation in the light. Plant Cell 10:1623-1636

Strain HH, Cope BT, Svec WA (1971) Analytical procedures for the isolation, identification, estimation and investigation of the chlorophylls. Methods Enzymol 23:452-476

Suzuki T, Kunieda T, Murai F, Morioka S, Shioi Y (2005) Mgdechelation activity in radish cotyledons with artificial and native substrates, Mg-chlorophyllin $a$ and chlorophyllide $a$. Plant Physiol Biochem 43:459-464

Thomas H (1987) Sid: a Mendelian locus controlling thylakoid membrane disassembly in senescing leaves of Festuca pratensis. Theor Appl Genet 73:551-555

Thomas H (1997) Chlorophyll: a symptom and a regulator of plastid development. New Phytol 136:163-181

Thomas H, Howarth CJ (2000) Five ways to stay green. J Exp Bot 51:329-337

Thomas H, Schellenberg M, Vicentini F, Matile P (1996) Gregor Mendel's green and yellow pea seeds. Bot Acta 109:3-4

Vicentini F, Hörtensteiner S, Schellenberg M, Thomas H, Matile P (1995) Chlorophyll breakdown in senescent leaves: identification of the biochemical lesion in a stay-green genotype of Festuca pratensis Huds. New Phytol 129:247-252

Wesley SV, Helliwell CA, Smith NA, Wang M, Rouse DT, Liu Q, Gooding PS, Singh SP, Abbot D, Stoutjesdijk PA, Robinson SP, Gleave AP, Green AG, Waterhouse PM (2001) Construct design for efficient, effective and high-throuput gene silencing in plants. Plant J 27:581-590

White OE (1916) Inheritance studies in Pisum I Inheritance of cotyledon color. Am Nat 50:530-547

Zelisko A, Garcia-Lorenzo M, Jackowski G, Jansson S, Funk C (2005) AtFtsH6 is involved in the degradation of the lightharvesting complex II during high-light acclimation and senescence. Proc Natl Acad Sci USA 102:13699-13704 